\title{
BH3-only proteins are dispensable for apoptosis induced by pharmacological inhibition of both $\mathrm{MCL}-1$ and $\mathrm{BCL}-\mathrm{X}_{\mathrm{L}}$
}

\author{
Georgia Greaves $^{1}$ - Mateus Milani ${ }^{1}$ - Michael Butterworth ${ }^{1} \cdot$ Rachel J. Carter $^{1}$ - Dominic P. Byrne ${ }^{2}$ Patrick A. Eyers ${ }^{2}$. \\ $\mathrm{Xu} \mathrm{Luo}{ }^{3} \cdot$ Gerald M. Cohen ${ }^{1,4} \cdot$ Shankar Varadarajan $^{1,4}$
}

Received: 14 May 2018 / Revised: 23 July 2018 / Accepted: 24 July 2018 / Published online: 5 September 2018

(c) The Author(s) 2018. This article is published with open access

\begin{abstract}
The impressive selectivity and efficacy of BH3 mimetics for treating cancer has largely been limited to BCL-2 dependent hematological malignancies. Most solid tumors depend on other anti-apoptotic proteins, including MCL-1, for survival. The recent description of S63845 as the first specific and potent MCL-1 inhibitor represents an important therapeutic advance, since MCL-1 is not targeted by the currently available BH3 mimetics, Navitoclax or Venetoclax, and is commonly associated with chemoresistance. In this study, we confirm a high binding affinity and selectivity of S63845 to induce apoptosis in MCL-1-dependent cancer cell lines. Furthermore, S63845 synergizes with other BH3 mimetics to induce apoptosis in cell lines derived from both hematological and solid tumors. Although the anti-apoptotic BCL-2 family members in these cell lines interact with a spectrum of pro-apoptotic BH3-only proteins to regulate apoptosis, these interactions alone do not explain the relative sensitivities of these cell lines to BH3 mimetic-induced apoptosis. These findings necessitated further investigation into the requirement of BH3-only proteins in BH3 mimetic-mediated apoptosis. Concurrent inhibition of BCL- $\mathrm{X}_{\mathrm{L}}$ and MCL-1 by BH3 mimetics in colorectal HCT116 cells induced apoptosis in a BAX-but not BAK-dependent manner. Remarkably this apoptosis was independent of all known BH3-only proteins. Although BH3only proteins were required for apoptosis induced as a result of BCL- $\mathrm{X}_{\mathrm{L}}$ inhibition, this requirement was overcome when both BCL- $\mathrm{X}_{\mathrm{L}}$ and MCL-1 were inhibited, implicating distinct mechanisms by which different anti-apoptotic BCL-2 family members may regulate apoptosis in cancer.
\end{abstract}

Edited by D. Vaux

Electronic supplementary material The online version of this article (https://doi.org/10.1038/s41418-018-0183-7) contains supplementary material, which is available to authorized users.

Shankar Varadarajan

svar@liverpool.ac.uk

1 Departments of Molecular and Clinical Cancer Medicine, Institute of Translational Medicine, University of Liverpool, Liverpool, Ashton Street, Liverpool L69 3GE, UK

2 Department of Biochemistry, Institute of Integrative Biology, Crown Street, Liverpool L69 7ZB, UK

3 Eppley Institute for Research in Cancer and Allied Diseases, Fred and Pamela Buffett Cancer Center, University of Nebraska Medical Center, Omaha, Nebraska 68198, USA

4 Departments of Molecular and Clinical Pharmacology, Institute of Translational Medicine, University of Liverpool, Liverpool, Ashton Street, Liverpool L69 3GE, UK

\section{Introduction}

Abnormal cell survival through resistance to apoptosis is a cardinal feature of most malignancies and plays a key role in chemoresistance. $[1,2]$ As the major regulators of the intrinsic mitochondrial pathway of apoptosis, the prosurvival BCL-2 family proteins (BCL-2, BCL- $\mathrm{X}_{\mathrm{L}}$, MCL1 , and BCL2A1) are attractive targets for novel cancer therapeutics. [3, 4] These proteins are proposed to function by binding and sequestering the pro-apoptotic BH3-only proteins, which in turn prevents the effector proteins, BAX and BAK, from forming pores in the mitochondrial membrane. The development of inhibitors of the pro-survival BCL-2 family proteins has proven particularly difficult because their inhibition requires disruption of these proteinprotein interactions. However, after many years of research, small molecule inhibitors known as $\mathrm{BH} 3$ mimetics, which target the anti-apoptotic BCL-2 family have now been developed. The first clinically approved $\mathrm{BH} 3$ mimetic was ABT-263 (Navitoclax), an orally available form of 
ABT-737, which inhibits BCL-2, BCL-X ${ }_{L}$, and BCL-w [3, 5-7] and potently induces apoptosis in several solid and hematological cancers. This was followed by the introduction of a BCL-2-specific inhibitor, ABT-199 (Venetoclax), which has received approval for treating refractory chronic lymphocytic leukemia (CLL), where BCL-2 addiction is a key feature in the pathogenesis of the disease. [8-10] Recently, BH3 mimetics specifically targeting BCL-X $\mathrm{L}_{\mathrm{L}}$ (A1331852) [11-15] and MCL-1 (A-1210477 and S63845) [1, $12,16]$ have also been developed.

In this study, we demonstrate that S63845 is a highly potent and selective MCL-1 inhibitor, which can synergize with other BH3 mimetics, including ABT-199 and A1331852 , to induce apoptosis in a wide range of cell lines derived from different hematological malignancies and solid tumors. Furthermore, we demonstrate that BH3-only proteins are required for apoptosis induced following BCL$\mathrm{X}_{\mathrm{L}}$ inhibition, whereas dual inhibition of $B C L-\mathrm{X}_{\mathrm{L}}$ and MCL-1 resulted in a $\mathrm{BH} 3$-only protein-independent cell death.

\section{Materials and methods}

\section{Cell culture}

Cell lines derived from mantle cell lymphoma (MAVER-1), diffuse large B cell lymphoma (U-2946), chronic myeloid leukemia (K562), acute myeloid leukemia (MOLM-13, OCI-AML-3, HL-60, MV-4-11, THP-1 and U-937), JurkatT-lymphocyte cell lines, wild-type and deficient in caspase9 (from Prof. J. Borst, The Netherlands Cancer InstituteAntoni van Leeuwenhoek, Amsterdam), caspase-8 and FADD (from Prof. J. Blenis, Weill Cornell Medicine, USA), non-small cell lung carcinoma (H1299), prostate cancer (PC-3, from Prof. Y. Ke, University of Liverpool, UK) and triple negative breast cancer (MDA-MB-231 from P. Meier, Institute of Cancer Research, London, UK) were cultured in RPMI 1640 medium. H929, a multiple myeloma cell line, was cultured in the same medium supplemented with $0.02 \%$ 2-mercaptoethanol. The non-small cell lung carcinoma cell line A549 was cultured in DMEM/ F12 supplemented with $1 \%$ non-essential amino acids. The pancreatic cancer cell line, SUIT-2 (from A. Mielgo, University of Liverpool, UK) was cultured in DMEM. Colon cancer cell lines HT-29 (from J. Parsons, University of Liverpool, UK) and HCT116 (wild-type, DKO and 8KO) [3, 17-22] as well as HCT116 cells deficient in BAX or BAK (from R. J. Youle, National Institute of Health, USA), were cultured in McCoys $5 \mathrm{~A}$ Modified media. All cell lines, unless otherwise specified, were either from DMSZ (Braunshweig, Germany) or ATCC (Middlesex, UK) and were validated using short tandem repeat profiling. Peripheral blood samples from CLL patients were obtained with patient consent and local ethics committee approval and cultured as described. [23] All media were supplemented with $10 \%$ fetal calf serum (Life Technologies Inc., Paisley, UK).

\section{Reagents}

A-1331852 and A-1210477 from AbbVie Inc. (North Chicago, IL, USA), S63845 from ActiveBiochem (New Jersey, USA), and ABT-199 and Z-VAD.FMK from Selleck Chemicals Co. (Houston, TX, USA) were used. Antibodies against BCL-X $\mathrm{L}_{\mathrm{L}}, \mathrm{BIM}$ and BAD from Cell Signalling Technology (MA, USA), NOXA from Millipore (Watford, UK), BID and tubulin from Abcam (Cambridge, UK), MCL-1, BCL-2 and PUMA from Santa Cruz Biotechnology (Santa Cruz, CA, USA) and BAX and cytochrome $c$ (BD BioSciences, California) were used for immunoblotting. Antibodies used for immunoprecipitation were MCL-1 (Y-37) and BCL-X $\mathrm{L}_{\mathrm{L}}$ from Abcam, BCL-2 from $\mathrm{BD}$ Biosciences and BAX (NT) from Merck-Millipore (Burlington, MA, USA). siRNAs specific to BCL-X (SI03025141), MCL-1 (SI02781205) and a non-targeting control (1027310) from Qiagen (Cambridge, UK) were incubated with Interferin siRNA transfection reagent (Polyplus transfection Inc., NY, USA) and added to cells at a concentration of $10 \mathrm{nM}$ for $72 \mathrm{~h}$.

\section{Differential scanning fluorimetry assay}

Thermal shift assays were performed using a StepOnePlus Real-Time PCR machine (Life Technologies, Paisley, UK) with Spyro-Orange dye (Invitrogen, Paisley, UK) and purified recombinant BCL-2 (Abcam) and MCL-1 protein, as previously described. [3]

\section{Cytochrome $c$ release, BAX translocation and apoptosis measurements}

To quantitate cytochrome $c$ release and BAX translocation, cells were grown on coverslips and treated with the specified inhibitors following a $0.5 \mathrm{~h}$ pre-treatment with the pancaspase inhibitor Z-VAD.FMK $(30 \mu \mathrm{M})$, and then fixed with $4 \%(\mathrm{v} / \mathrm{v})$ paraformaldehyde and permeabilized with $0.5 \%(\mathrm{v} / \mathrm{v})$ Triton X-100 in PBS, followed by incubation with primary and fluorophore-conjugated secondary antibodies prior to visualization under a fluorescent microscope. The extent of cytochrome $c$ released from the mitochondria or BAX translocation to the mitochondria was quantified by counting at least 300 cells from three independent experiments. The extent of apoptosis in cells following different 
treatments was quantified using an Attune NxT flow cytometer (Thermo Fisher Scientific, Paisley, UK), as previously described. [24]

\section{Gel filtration, immunoprecipitation and Western blotting}

Recombinant MCL-1 purification, size exclusion chromatography and immunoprecipitation experiments were carried out as previously described. [3, 25] Western blotting was carried out according to standard protocols. Briefly, $50 \mu \mathrm{g}$ of total protein lysate was subjected to SDS-PAGE electrophoresis. Subsequently proteins were transferred to nitrocellulose membranes and protein bands visualized with ECL reagents (GE Healthcare).

\section{Clonogenic studies}

Cells were seeded in 6 well plates at a density of 2000 cells/ well and exposed to the specified inhibitors (100 nM) $24 \mathrm{~h}$ post-seeding. Cells were fixed on day 7 using methanol and acetic acid and then stained with crystal violet and colonies counted using the GelCount tumor colony counter (Oxford Optronix, Abbingdon, UK).

\section{Statistics}

For time-course studies, a two-way ANOVA was performed using Dunnet's multiple comparisons analysis and other studies were analyzed for statistical significance with oneway ANOVA using Sidak's multiple comparisons analysis. The asterisks depicted correspond to the following $p$ values: $* p \leq 0.05 ; * * p \leq 0.01 ; * * * p \leq 0.001$.

\section{Results}

\section{S63845 is a potent MCL-1 inhibitor and weak inducer of apoptosis in BCL-2- but not $B C L-X_{L}$-dependent cells}

Consistent with previous findings, [1] we demonstrated that S63845 is a potent MCL-1 inhibitor when compared with A1210477 , as assessed by a concentration-dependent thermal stabilization of MCL-1 in our in vitro thermal shift assay [3] (Fig. 1a). S63845 also induced rapid apoptosis in two MCL1-dependent cell lines (H929 and U-2946), [3, 6] with an $\mathrm{IC}_{50} \sim 100 \mathrm{nM}$, demonstrating $\sim 100$-fold higher potency than A-1210477 (Fig. 1b). To assess the specificity of S63845 to a

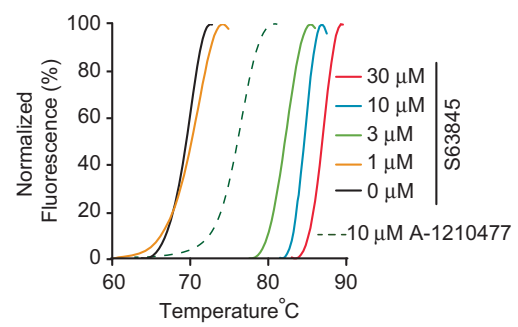

d

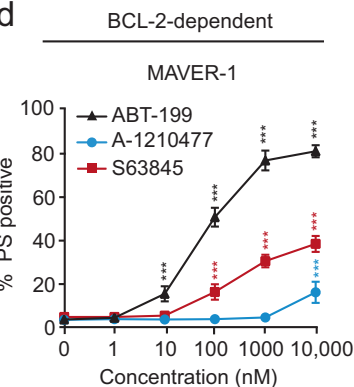

e

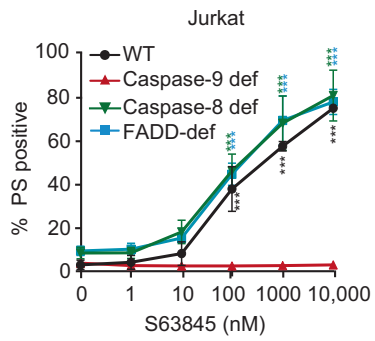

Fig. 1 S63845 binds MCL-1 and induces apoptosis in several cancer cell lines. a S63845 exhibits a concentration-dependent shift in MCL-1 stabilization in a thermal stability assay. b S63845 and A-1210477 exhibit concentration-dependent apoptosis, as assessed by PS externalization using flow cytometry, in MCL-1-dependent cell lines, H929 and U-2946 after $4 \mathrm{~h}$ of exposure. c S63845 and A-1210477 fail to induce apoptosis in a BCL- $\mathrm{X}_{\mathrm{L}}$-dependent cell line, $\mathrm{K} 562$, at $4 \mathrm{~h}$, unlike A-1331852 (positive control). d MAVER-1, a BCL-2 dependent cell line, when exposed to the indicated $\mathrm{BH} 3$ mimetics for $4 \mathrm{~h}$ resulted in
MCL-1-dependent

U-2946
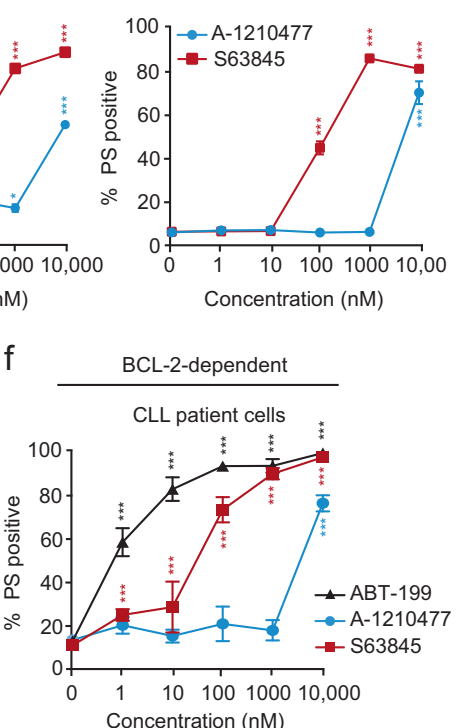

BCL-X - dependent

K562

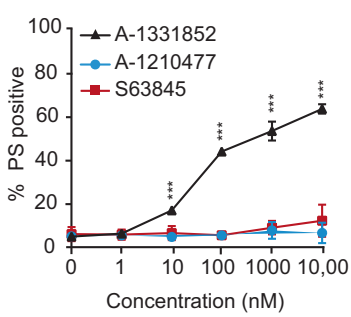

g

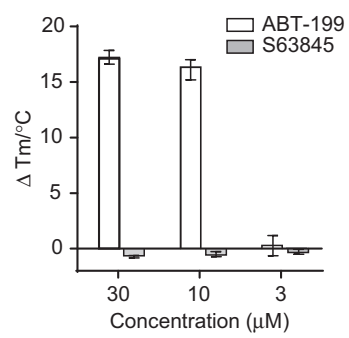

enhanced apoptosis. e Jurkat cells (wild type and deficient in the specified proteins), exposed to increasing concentrations of S63845 for $24 \mathrm{~h}$ exhibited caspase-9-dependent apoptosis. f Primary cells isolated from CLL patients, exposed to the indicated BH3 mimetics for $8 \mathrm{~h}$, exhibited varying extents of apoptosis. g ABT-199 but not S63845 exhibits a concentration-dependent shift in BCL-2 stabilization in a thermal stability assay. Error bars $=$ Mean \pm SEM (standard error of the mean); $* p \leq 0.05 ; * * p \leq 0.01 ; * * * p \leq 0.001$ 
induce apoptosis in a MCL-1 dependent manner, we exposed K562 cells (a BCL- $\mathrm{X}_{\mathrm{L}}$-dependent cell line) and MAVER-1 cells (a BCL-2-dependent cell line) to increasing concentrations of S63845 (Fig. 1c, d). As expected, S63845 (as well as A-1210477) failed to induce apoptosis in K562 cells, whereas extensive apoptosis was observed following exposure to A-1331852 (Fig. 1c). However, S63845 (as well as A-1210477) induced a concentration dependent increase in apoptosis in MAVER-1 cells, albeit at higher concentrations than ABT-199 (Fig. 1d), suggesting that S63845 could either induce death in a non-specific manner or that it could also be a weak inhibitor of BCL-2. To test this, we exposed Jurkat cells (wild type as well as deficient in FADD, caspase- 8 and caspase-9) to increasing concentrations of S63845. While S63845 induced similar levels of apoptosis in wild type as well as FADD/ caspase-8 deficient Jurkat cells, cells deficient in caspase-9 were completely resistant to S63845-mediated apoptosis (Fig. 1e), thus demonstrating that $\mathrm{S} 63845$ both specifically induced the intrinsic pathway of apoptosis and also did not induce non-specific cell death. To identify whether S63845, in addition to binding and inhibiting MCL-1, can also potentially bind and inhibit BCL-2, we exposed primary CLL cells that exclusively depend on BCL-2 for survival, [8] to increasing concentrations of S63845 and A-1210477. While ABT-199 (positive control) induced apoptosis in these cells at low nanomolar concentrations, both S63845 and A-1210477 also induced significant apoptosis at concentrations similar to their $\mathrm{IC}_{50}$ values in MCL-1 dependent cells (Fig. 1f). However, we did not detect any concentration-dependent thermal stabilization of BCL-2 following S63845 in an in vitro thermal shift assay, which was in marked contrast to the thermal stabilization of BCL-2 following ABT-199 in this assay (Fig. 1g). Taken together, our data demonstrates that under these experimental conditions, S63845 does not bind BCL-2 and induces apoptosis in a MCL-1-specific manner.

\section{S63845 is more potent than ABT-199 in inducing apoptosis in AML cell lines}

Recent reports indicate that cells derived from acute myeloid leukemia (AML) patients can be effectively targeted with a BCL-2 and/or MCL-1 inhibitor, [11, 13-15] although other studies suggest that these cells depend primarily on BCL-2 for survival. [16] Indeed, MCL-1 inhibitors are currently entering clinical trials to treat AML and multiple myeloma patients (Clinical trials-NCT02979366; NCT02675452; NCT02992483). Therefore, we investigated whether S63845 alone or in combination with ABT-199 could induce apoptosis in a panel of AML cell lines. Of the six cell lines tested, MV-4-11 cells exhibited high sensitivity to both S63845 (IC $50 \sim 20 \mathrm{nM})$ and ABT-199 $\left(\mathrm{IC}_{50} \sim\right.$ 50-100 nM) individually. THP-1 was much more resistant to ABT-199 but sensitive to $\mathrm{S} 63845$ ( $\mathrm{IC}_{50} \sim 50 \mathrm{nM}$ ). OCIAML-3, U937 and MOLM-13 were completely resistant to ABT-199 but somewhat sensitive to S63845 at higher concentrations. In contrast, HL-60 cells were largely resistant to both BH3 mimetics individually (Fig. 2a). In the resistant cell lines, particularly U-937 and HL-60, BCL-2 and $\mathrm{BCL}-\mathrm{X}_{\mathrm{L}}$ appeared to perform redundant functions in cell survival, whereas MOLM-13 cells appeared to depend on BCL- $\mathrm{X}_{\mathrm{L}}$ for survival, as exposure to A-1331852 alone resulted in significant apoptosis (Supplemental Fig. S1). Nevertheless, all AML cells examined were more sensitive to the combination of S63845 and ABT-199 (Fig. 2a).

\section{Interactions between the pro and anti-apoptotic BCL-2 family members do not reflect the relative sensitivities of $A M L$ cell lines to undergo $B H 3$ mimetic-mediated apoptosis}

BH3 mimetic-induced apoptosis is usually attributed to displacement of BH3-only proteins from their anti-apoptotic partners, leading to apoptosis. Since the different AML cell lines exhibited varied responses to $\mathrm{BH} 3$ mimetic-mediated apoptosis, we wished to assess if this could be attributed to altered binding and/or displacement of the different BH3only proteins to their known survival counterparts. Therefore, we immunoprecipitated the anti-apoptotic proteins required for survival and probed for each of the BH3-only proteins (Fig. 2b-d). In MV-4-11 cells, which are sensitive to both ABT-199 and S63845, BIM and PUMA bound to BCL-2 and were slightly displaced following exposure to ABT-199 (Fig. 2b). In contrast, the traces of BIM and NOXA bound to MCL-1 in these cells were readily displaced after S63845 exposure, in-line with the extent of apoptosis observed with $\mathrm{S} 63845\left(\mathrm{IC}_{50} \sim 20 \mathrm{nM}\right)$. Neither BID nor BAD interacted with MCL-1 or BCL-2 in these cells (Fig. 2b). In OCI-AML-3, exposure to ABT-199 also resulted in a partial displacement of BIM and PUMA from BCL-2, despite the cells being largely resistant to ABT-199 (Fig. 2c). Similar to MV-4-11, BIM and NOXA bound to MCL-1 were readily displaced with S63845, and BID and BAD failed to interact with either MCL-1 or BCL-2 (Fig. 2c). In the resistant HL-60 cell line, BIM and PUMA bound to BCL-2 was not displaced following ABT-199, compatible with the cells being largely resistant to ABT-199 (Fig. 2d). BID and BAD were not bound to either MCL-1 or BCL-2. Moreover, of all the BH3-only members, only traces of NOXA appeared to interact with MCL-1 and was displaced following S63845 (Fig. 2d). Taken together, these results suggest that interactions between the pro- and antiapoptotic BCL-2 family members may not necessarily reflect the relative sensitivities of different AML cell lines to undergo $\mathrm{BH} 3$ mimetic-mediated apoptosis. 

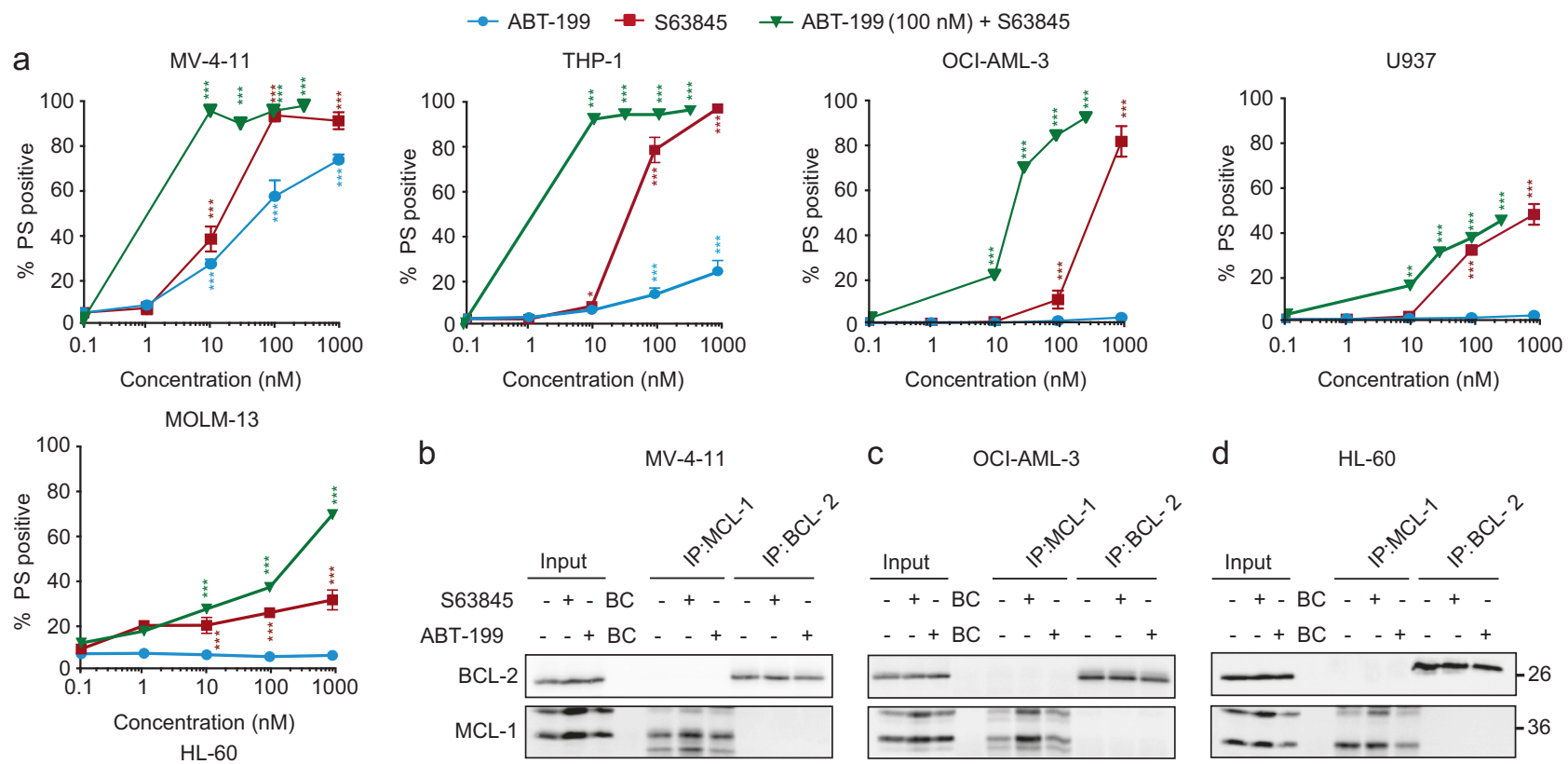

b

OCI-AML-3

d
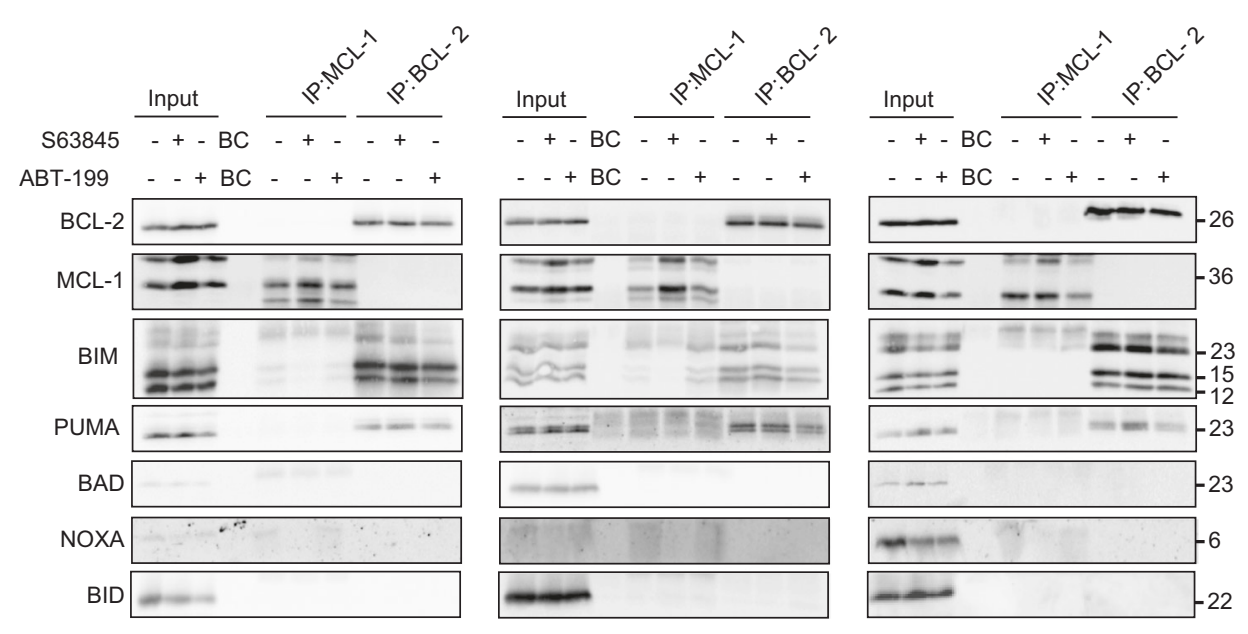

Fig. 2 S63845 can synergize with ABT-199 to induce apoptosis in AML cell lines. a AML cell lines were exposed to a concentration range of S63845 with ABT-199 at a constant concentration of $100 \mathrm{nM}$ for $24 \mathrm{~h}$ and assessed for PS externalization. Immunoprecipitation of MCL-1 and BCL-2 was carried out in b MV-4-11, c OCI-AML-3, and d HL-60 cells, following exposure to S63845 (30 nM for MV-4-11 and

\section{Interactions of $B C L-X_{L}$ and MCL-1 with different BH3-only members differ in solid tumor cell lines}

Unlike several hematological malignancies, cancer cells derived from most solid tumors depend on both BCL- $\mathrm{X}_{\mathrm{L}}$ and MCL-1 for survival. [3, 17, 19-22] Exposure of cells derived from a panel of solid tumors, including non-small cell lung carcinoma (H1299 and A549), pancreas (SUIT2), colon (HCT116 and HT-29) and prostate cancer (PC3) to a combination of $\mathrm{S} 63845$ or A-1331852 resulted in a marked induction of apoptosis, confirming that these cell lines depend on both BCL- $\mathrm{X}_{\mathrm{L}}$ and MCL-1 for survival (Fig. 3a). To corroborate earlier findings in AML cell lines (Fig. 2) in non-hematological cells, we repeated immunoprecipitation studies and assessed interactions between the different BH3-only proteins with their key pro-survival counterparts, namely BCL- $\mathrm{X}_{\mathrm{L}}$ and MCL-1. In $\mathrm{H} 1299$ cells, BIM and PUMA were preferentially bound to MCL-1 and BCL- $\mathrm{X}_{\mathrm{L}}$, respectively, and were readily displaced following exposure to the relevant $\mathrm{BH} 3$
$100 \mathrm{nM}$ for the other 2 cell lines) or ABT-199 (50 nM for MV-4-11 and $100 \mathrm{nM}$ for the other 2 cell lines) for $6 \mathrm{~h}$ (MV-4-11) or $8 \mathrm{~h}$ (OCIAML-3 and HL60). The eluted complexes were immunoblotted for the indicated proteins. The input represents the cell lysates and $\mathrm{BC}$, beads control. Error bars $=$ Mean \pm SEM; ${ }^{*} p \leq 0.05 ; * * p \leq 0.01 ; * * * p \leq 0.001$

mimetics (Fig. 3b). This was particularly evident following exposure to S63845, in which the newly released BIM from the MCL-1 complex exhibited enhanced binding to $\mathrm{BCL}-\mathrm{X}_{\mathrm{L}}$. Similarly, exposure to A-1331852 displaced BIM from BCL- $\mathrm{X}_{\mathrm{L}}$, which in turn facilitated its binding to MCL-1 (Fig. 3b). Other BH3-only proteins like NOXA and BAD exhibited selectivity in binding to MCL-1 and BCL- $\mathrm{X}_{\mathrm{L}}$, respectively and were also displaced after exposure to the relevant $\mathrm{BH} 3$ mimetics. No detectable binding of BID to either BCL- $\mathrm{X}_{\mathrm{L}}$ or MCL-1 was observed (Fig. 3b). In SUIT-2 cells, BIM was bound to both MCL-1 and BCL- $\mathrm{X}_{\mathrm{L}}$ and was displaced to differing extents with the specific $\mathrm{BH} 3$ mimetics (Fig. 3c). In contrast to H1299, PUMA was not bound to either MCL-1 or BCL- $\mathrm{X}_{\mathrm{L}}$ (Fig. 3c). However, there were some similarities, especially with NOXA being bound to MCL-1 and displaced with S63845, and BAD bound to BCL- $\mathrm{X}_{\mathrm{L}}$, which could be disrupted following A-1331852 (Fig. 3c). Therefore, H1299 and SUIT-2 cells exhibit significant differences in their BCL-2 family interaction 

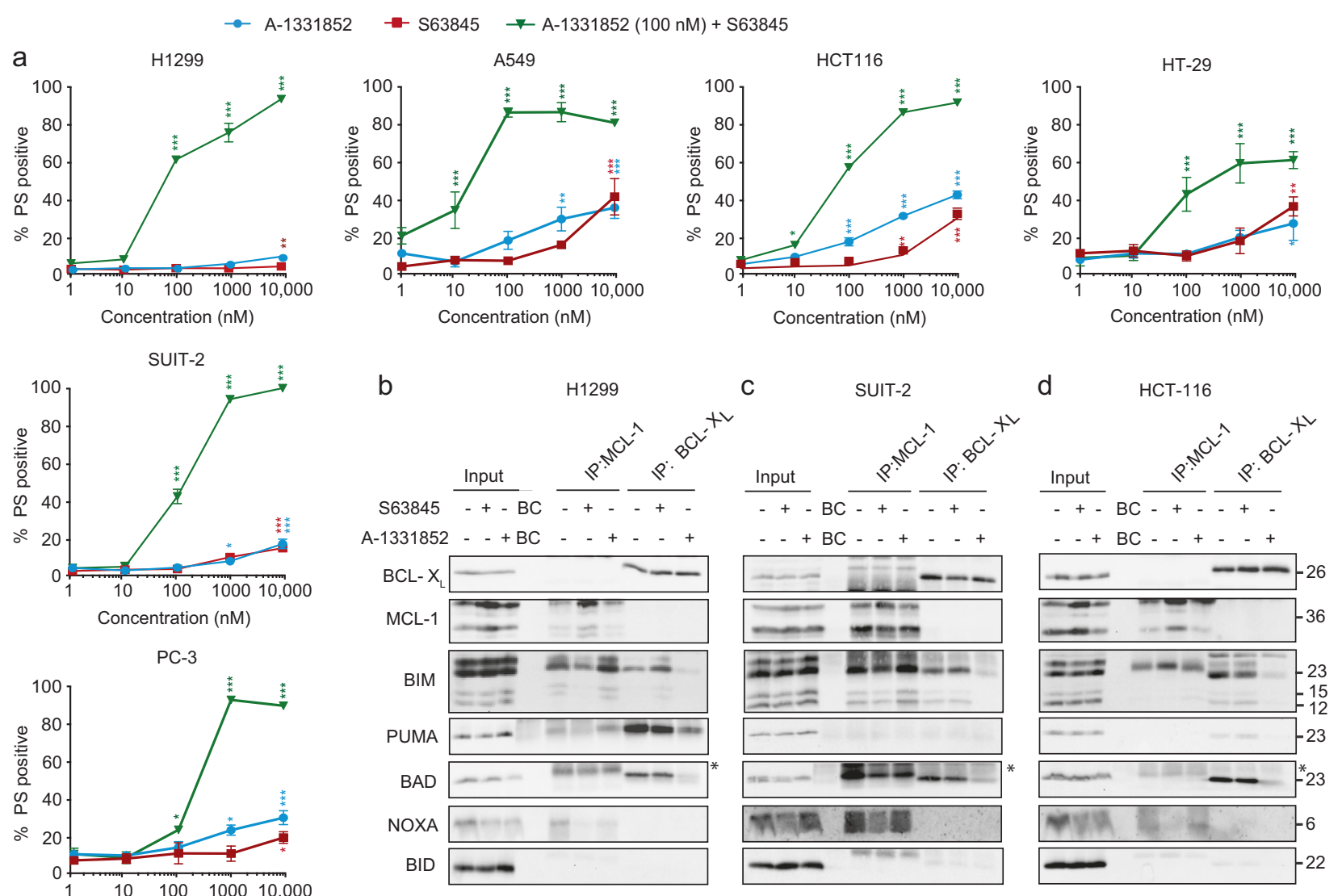

Fig. $3 \mathrm{BH} 3$-only proteins have different binding preferences in various solid tumor cell lines despite similar sensitivities to BH3 mimetics. a Solid tumor cell lines were treated with a concentration range of S63845 and a constant concentration of $100 \mathrm{nM}$ of A-1331852 for $4 \mathrm{~h}$ and assessed for PS externalization. Immunoprecipitation of MCL-1

profile, despite similar expression levels of the different proteins and cell death responsiveness to the combination of A-1331852 and S63845. This was even more evident in HCT116 cells, in which most of the BH3-only proteins, with the exception of NOXA, interacted exclusively with $\mathrm{BCL}-\mathrm{X}_{\mathrm{L}}$, and could be readily displaced from their binding partners with A-1331852 (Fig. 3d). Collectively, these results suggest that the preferential sequestration of different $\mathrm{BH} 3$-only proteins to distinct anti-apoptotic counterparts does not solely dictate dependency of a cell line on an individual anti-apoptotic protein.

\section{BH3-only proteins are dispensable for $\mathrm{BH} 3$ mimetic-induced apoptosis in HCT116 cells}

Since BH3-only proteins have recently been demonstrated to be dispensable for cell death, $[18,26]$ we evaluated whether selective inhibitors of $B C L-X_{L}$ and MCL-1 could induce apoptosis in the absence of all and BCL-X $\mathrm{X}_{\mathrm{L}}$ was carried out in H1299 b, SUIT-2 c, and HCT116 d cells exposed to $\mathrm{S} 63845(100 \mathrm{nM})$ or A-1331852 $(100 \mathrm{nM})$ for $2 \mathrm{~h}$, and the eluted complexes were immunoblotted for the indicated proteins. $*$ in the blots denotes non-specific bands. Error bars $=$ Mean \pm SEM (standard error of the mean); $* p \leq 0.05 ; * * p \leq 0.01 ; * * * p \leq 0.001$

known BH3-only proteins. Remarkably, in HCT116 8KO cells, which lack the 8 key BH3-only proteins, BIM, BID, PUMA, NOXA, HRK, BMF, BIK and BAD, [18] a combination of S63845 and A-1331852 induced apoptosis to the same extent as that of wild-type (WT) HCT116 cells (Fig. 4a). Furthermore, this combination of BH3 mimetics resulted in a BAX-dependent but BAKindependent apoptosis in these cells (Fig. 4b), emphasizing a crucial role for BAX in BH3 mimetic-mediated apoptosis. This raised the possibility of BAX directly interacting with $\mathrm{BCL}-\mathrm{X}_{\mathrm{L}}$ and/or MCL-1 to antagonize apoptosis. To test this hypothesis, we immunoprecipitated MCL-1 and BCL- $\mathrm{X}_{\mathrm{L}}$ complexes from both HCT116 WT and $8 \mathrm{KO}$ cells but failed to detect BAX interaction with either anti-apoptotic protein (Fig. 4c). As a positive control, we immunoprecipitated active-BAX in these lysates, which pulled down BAX only following treatment with a combination of S63845 and A-1331852, indicative of BAX activation during apoptosis (Fig. 4c). These findings suggested that the $\mathrm{BH} 3$ mimetics could 


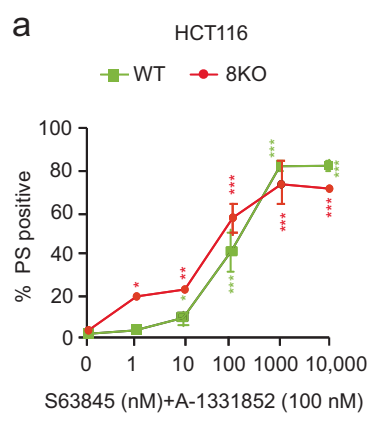

b

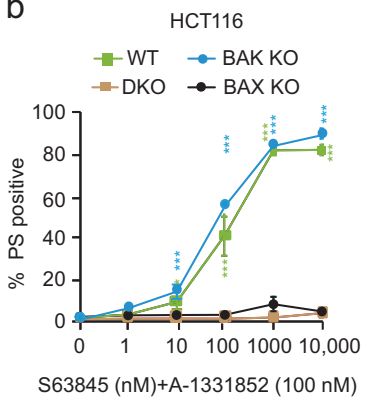

d

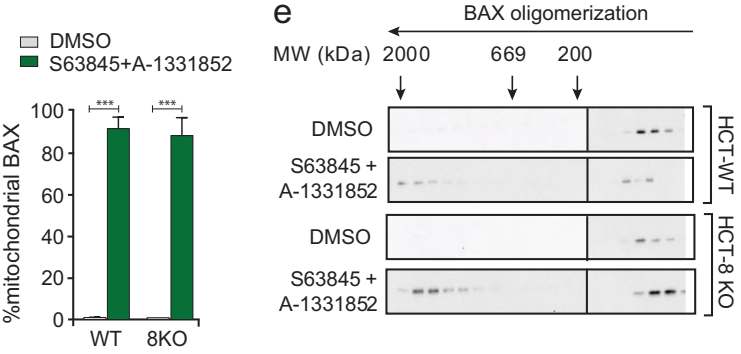

Fig. 4 BH3 mimetics induce apoptosis even in the absence of BH3only proteins in HCT116 cells. a HCT116 cells, wild-type (WT) and deficient in 8 different pro-apoptotic $\mathrm{BH} 3$-only proteins (8KO), were exposed to A-1331852 $(100 \mathrm{nM})$ in combination with increasing concentrations of S63845 for $4 \mathrm{~h}$ and assessed for PS externalization. b Same as (a) but the experiments were carried out with HCT116 WT cells or cells deficient in BAX, BAK or both (DKO). c Immunoprecipitation of MCL-1, BCL- $\mathrm{X}_{\mathrm{L}}$ and active-BAX in the indicated cells exposed to a combination of S63845 (100 nM) and A-1331852 $(100 \mathrm{nM})$, following a $0.5 \mathrm{~h}$ pre-treatment with z-VAD.FMK $(30 \mu \mathrm{M})$, were performed to assess BAX interaction. d The level of

have displaced other protein(s) distinct from the $8 \mathrm{BH}$ only proteins from BCL- $\mathrm{X}_{\mathrm{L}}$ and/or MCL-1, which in turn activated BAX to facilitate mitochondrial outer membrane permeabilization (MOMP) and apoptosis. Apoptosis induction in the $8 \mathrm{KO}$ cells was accompanied by translocation of BAX from the cytosol to the mitochondrial membrane (Fig. 4d), BAX oligomerization into high molecular mass complexes as assessed by gel filtration (Fig. 4e), and MOMP, measured by the release of mitochondrial cytochrome $c$ (Fig. 4f). Together, this negates a role for BH3-only proteins in several critical steps of the intrinsic apoptotic pathway induced by $\mathrm{BH} 3$ mimetics.

\section{Inhibition of $B C L-X_{L}$ and $M C L-1$ results in $\mathrm{BH} 3$-independent apoptosis}

Although many cell lines derived from solid tumors depend on both BCL- $\mathrm{X}_{\mathrm{L}}$ and MCL-1 for survival, our studies revealed that inhibition of $\mathrm{BCL}-\mathrm{X}_{\mathrm{L}}$ following exposure to A-1331852 was sufficient to induce significant apoptosis in a panel of cell lines (Fig. 5a). To assess whether A1331852-mediated apoptosis also occurred in the absence of
C HCT116 WT HCT116 8KO

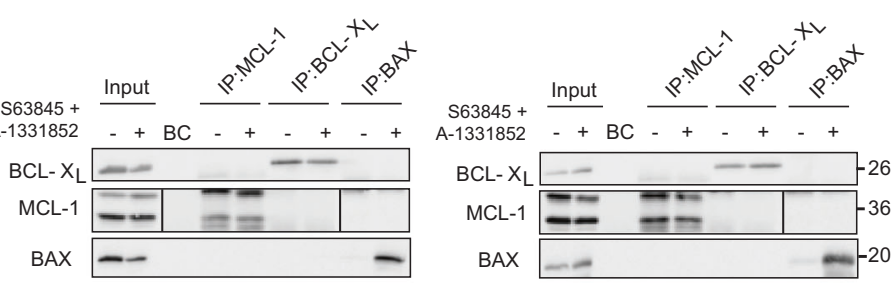

C $\square$ DMSO
$\square$ S63845+A-1331852
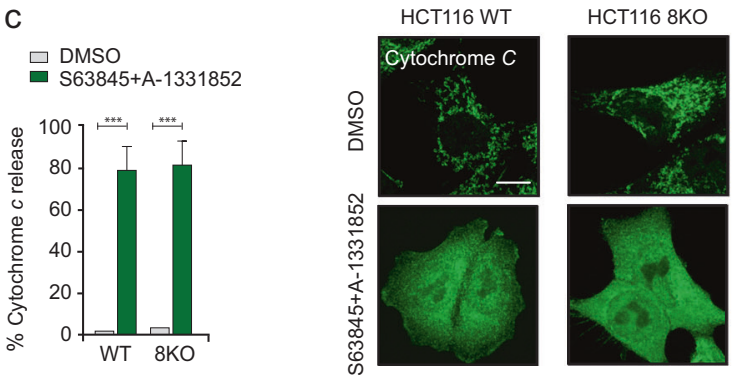

mitochondrial BAX in WT and 8KO cells following S63845 and A1331852 treatment (both $100 \mathrm{nM}$ ) for $4 \mathrm{~h}$ was assessed by immunocytochemistry using an anti-BAX antibody. e Western blots of different molecular weight fractions from FPLC showing BAX oligomerization in HCT116 WT and 8KO cells upon exposure to S63845 (100 nM) and A-1331852 (100 nM) for $2 \mathrm{~h}$. f Quantification and representative images of cytochrome $c$ release in the indicated cell lines, from three independent experiments, following exposure to S63845 $(100 \mathrm{nM})$ and A-1331852 $(100 \mathrm{nM})$ for $4 \mathrm{~h}$ in the indicated cells. Error bars $=$ Mean \pm SEM (standard error of the mean); $* p \leq$ $0.05 ; * * p \leq 0.01 ; * * * p \leq 0.001$

all known BH3-only proteins, we employed HCT116 WT and $8 \mathrm{KO}$ cells to assess several hallmarks of apoptosis following BCL- $\mathrm{X}_{\mathrm{L}}$ inhibition. Strikingly, exposure to A1331852, but not S63845, resulted in enhanced apoptosis, as assessed by phosphatidylserine (PS) externalization, MOMP and mitochondrial translocation of BAX in HCT116 WT but not $8 \mathrm{KO}$ cells (Fig. 5b-d) suggesting that A-1331852-mediated apoptosis requires the presence of BH3-only proteins. Support for this suggestion was also provided by the enhanced apoptosis observed in WT, but not $8 \mathrm{KO}$, cells following siRNA knockdown of BCL- $\mathrm{X}_{\mathrm{L}}$ (Fig. 5e). However, this protective effect in $8 \mathrm{KO}$ cells was abolished when both BCL- $\mathrm{X}_{\mathrm{L}}$ and MCL-1 were downregulated, suggesting a requirement of $\mathrm{BH} 3$-only proteins in BCL- $\mathrm{X}_{\mathrm{L}}$ but not when both BCL- $\mathrm{X}_{\mathrm{L}}$ and MCL-1 were downregulated. Similarly, exposure to A-1331852 significantly reduced the surviving fraction of WT, but not $8 \mathrm{KO}$, cell populations (Fig. 5f). As expected, S63845 had no effect on clonogenic survival in either of the cell types, while a combination of the two $\mathrm{BH} 3$ mimetics obliterated clonogenicity in both cell types (Fig. 5f). Taken together, our data convincingly argue against the requirement of 

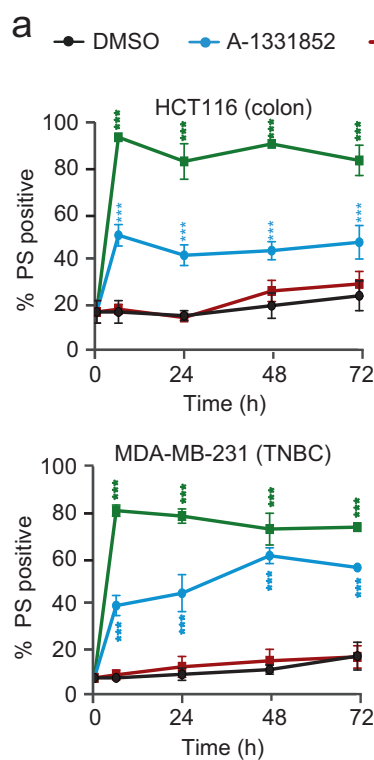

e

$$
\square \text { HCT116 WT }
$$

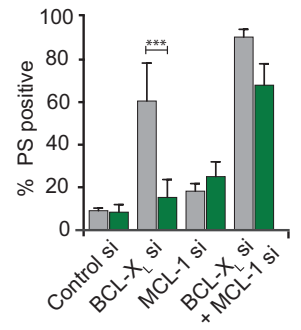

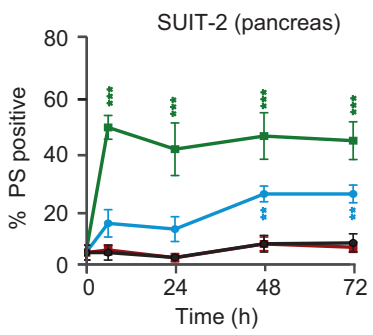

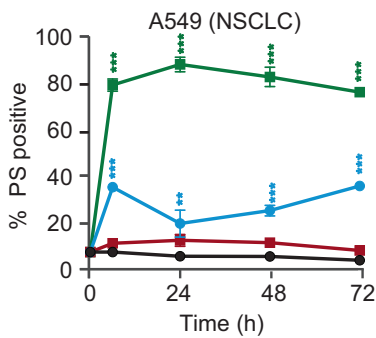

f $\square$ HCT116 WT $\square$ HCT116 8KO ***

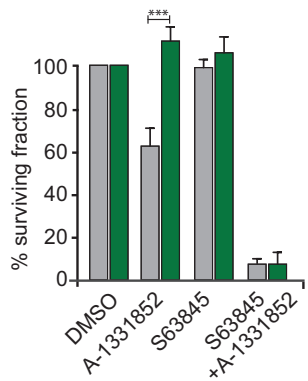

b

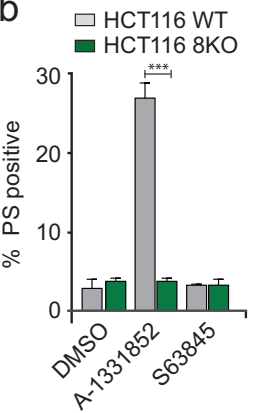

d
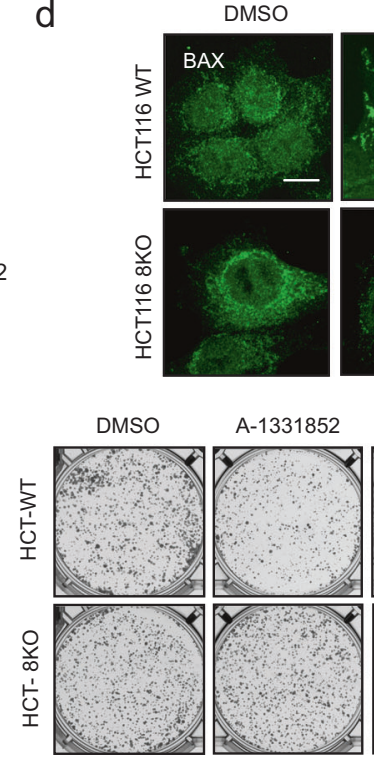

A-1331852
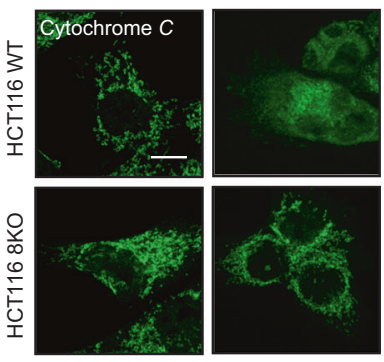

S63845
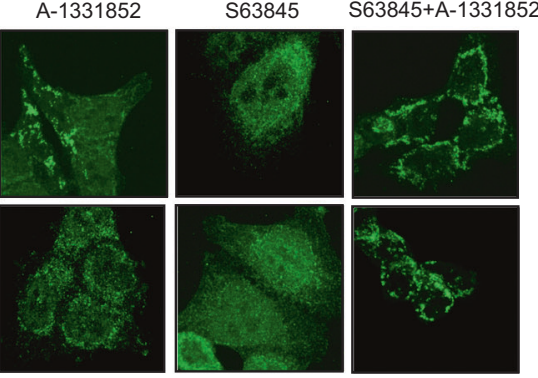

Fig. 5 Apoptosis regulated by $\mathrm{BCL}-\mathrm{X}_{\mathrm{L}}$ requires $\mathrm{BH} 3$-only proteins. a Apoptosis was assessed by PS externalization in the indicated cell lines following exposure to A-1331852 (100 nM) and/or S63845 (100 $\mathrm{nM}$ ) for the indicated times. HCT116 WT and 8KO cells were exposed to S63845 or A-1331852 $(100 \mathrm{nM})$ for $24 \mathrm{~h}$ and assessed for $\mathbf{b}$ PS externalization, cytochrome $c$ release and $\mathbf{d}$ BAX translocation. For (c) and (d), cells were pre-treated with z-VAD.FMK $(30 \mu \mathrm{M})$ for $0.5 \mathrm{~h}$ before exposure to $\mathrm{BH} 3$ mimetics. HCT116 WT and $8 \mathrm{KO}$ cells were e

BH3-only proteins in apoptosis regulated by a combination of both BCL-X $\mathrm{L}_{\mathrm{L}}$ and MCL-1 (Fig. 6).

\section{Discussion}

Prior to the discovery of S63845, putative MCL-1 inhibitors were poorly suited for clinical evaluation because they either lacked potency (high micromolar concentrations were required to inhibit MCL-1) or specificity resulting in undesirable toxicity. [3, 24, 27, 28] However, the development of $\mathrm{S} 63845$ potentially strengthens the use of a wide variety of $\mathrm{BH} 3$ mimetics in cancer therapy. In our hands, S63845 bound MCL-1 more extensively than A-1210477 and resulted in marked

transfected with siRNA against BCL- $\mathrm{X}_{\mathrm{L}}$ and/or MCL-1 for $72 \mathrm{~h}$ and assessed for PS externalization or $\mathbf{f}$ exposed to A-1331852 (100 nM) and/or S63845 (100 nM) and the number of colonies formed after $7 \mathrm{~d}$ was counted and images taken using GelCount. Representative images from the clonogenic assay are shown in the right panel. Error bars = Mean \pm SEM (standard error of the mean); $* \mathrm{p} \leq 0.05$; $* * \mathrm{p} \leq 0.01$; $* * * \mathrm{p} \leq 0.001$

apoptosis in MCL-1-dependent cell lines (Fig. 1), and synergized with ABT-199 and A-1331852 to induce apoptosis in various cancer cell lines (Figs. 2 and 3), supporting the concept that a potent MCL-1 inhibitor, such as S63845, could be a valuable addition to the BH3 mimetic armamentarium.

Our finding that $\mathrm{BH} 3$-only proteins are dispensable for BH3 mimetic-mediated apoptosis in HCT116 cells is striking, particularly when the proposed mechanism of action of $\mathrm{BH} 3$ mimetics is taken into consideration (Fig. 4). Since apoptosis induction in these cells still required $\mathrm{BAX}$ and not $\mathrm{BAK}$, [29] it is possible that $\mathrm{BH} 3$ mimetics could indirectly activate $\mathrm{BAX}$ by disrupting the interaction of BAX with BCL-X $\mathrm{L}_{\mathrm{L}}$ and/or MCL-1. However, we could not detect any BAX interaction with either 


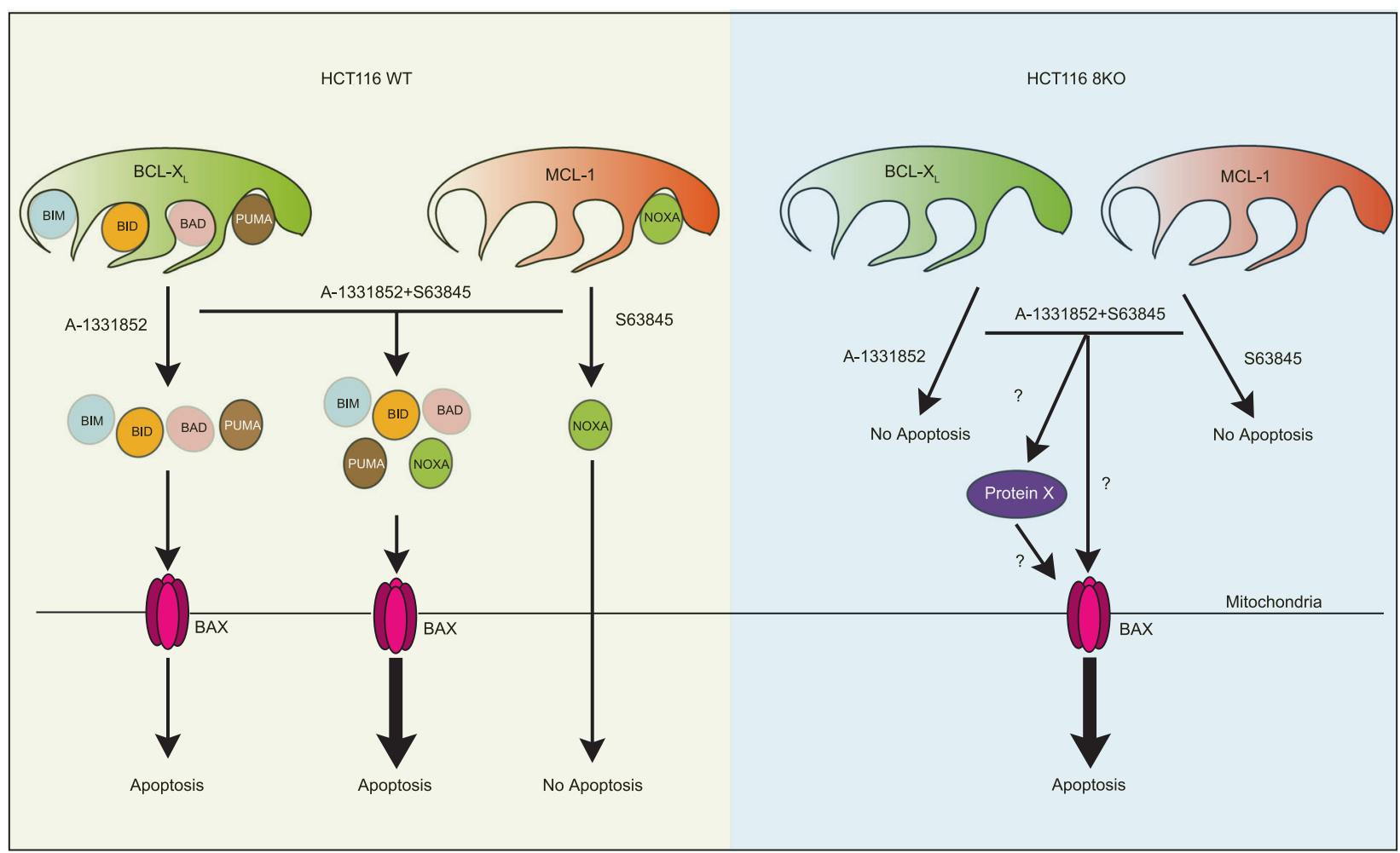

Fig. 6 BCL-2 family members differ in their dependence on proapoptotic BH3-only members to exert their anti-apoptotic functions. In HCT116 cells, multiple BH3-only proteins, such as BIM, BID, BAD and PUMA are sequestered by BCL- $\mathrm{X}_{\mathrm{L}}$, whereas NOXA is the only $\mathrm{BH} 3$-only protein bound to MCL-1. While the displacement of $\mathrm{BH} 3-$ only proteins from BCL- $\mathrm{X}_{\mathrm{L}}$ following A-1331852 is sufficient to induce apoptosis in these cells, displacement of NOXA from MCL-1 following S63845 failed to induce apoptosis. However, the combination of A-1331852 and S63845 released all the BH3-only proteins from the anti-apoptotic counterparts and resulted in pronounced apoptosis (bold arrow). In the absence of all eight $\mathrm{BH} 3$-only proteins, neither A-1331852 nor S63845 alone resulted in apoptosis. However, a combination of A-1331852 and S63845 still resulted in pronounced apoptosis, even in the absence of all $\mathrm{BH} 3$-only proteins. This could be due to the release of another pro-apoptotic $\mathrm{BH} 3$ domain-containing 'protein $\mathrm{x}$ ' from MCL-1 and/or BCL- $\mathrm{X}_{\mathrm{L}}$, which in turn could activate $\mathrm{BAX}$ in the $8 \mathrm{KO}$ cells. Alternatively, $\mathrm{BH} 3$ mimetics could indirectly result in the accumulation of BAX (either by inhibition of retrotranslocation or by passive diffusion, following the neutralization of the anti-apoptotic members) in the outer mitochondrial membrane, which in turn could result in BAX activation and apoptosis in the $8 \mathrm{KO}$ cells of these anti-apoptotic proteins, despite efficient mitochondrial translocation and oligomerization of BAX, which resulted in MOMP and PS externalization (Fig. 4). Failure to detect BAX in complex with anti-apoptotic proteins could possibly be due to the dynamic nature of this interaction. Although BAX is generally considered to translocate from the cytosol to the outer mitochondrial membrane following apoptotic stimuli, several reports suggest a role for the anti-apoptotic BCL-2 family members in the constitutive retrotranslocation of BAX from the mitochondrial outer membrane to the cytosol even under non-apoptotic conditions. [30-35] Neutralization of the anti-apoptotic BCL-2 family members with $\mathrm{BH} 3$ mimetics could markedly reduce BAX retrotranslocation to the cytosol, [33, 36] thus retaining it in the outer mitochondrial membrane, which may facilitate direct activation of BAX (Fig. 6). [18] This could explain the apoptosis observed following BH3 mimetics in HCT116 cells, even in the absence of all known BH3-only proteins (Fig. 4).

Our data reveal that the requirement for BH3-only proteins in BCL- $\mathrm{X}_{\mathrm{L}}$-regulated apoptosis can clearly be overcome when both MCL-1 and BCL- $\mathrm{X}_{\mathrm{L}}$ are inhibited simultaneously (Figs. 4 and 5). Recent reports have demonstrated novel interacting partners of MCL-1, such as the BH3 domain-containing SUFU [37] and VLCAD, [38] which regulate distinct cellular functions. Therefore, other BH3-domain containing proteins distinct from the 8 key BH3-only members could interact with MCL-1 and/or BCL$\mathrm{X}_{\mathrm{L}}$ and play a role in these events (Fig. 6). In summary, our results demonstrate that $\mathrm{BH} 3$ mimetics induce apoptosis even in the absence of the eight best characterized BH3-only proteins, while also identifying differences in the regulation of cell survival by the different anti-apoptotic BCL-2 family members. This highlights the need to understand the 
fundamental mechanisms of apoptosis in order to improve therapeutic approaches using BH3 mimetics.

Acknowledgements We thank AbbVie for providing A-1331852 and A-1210477. We thank Drs. Youle, Meier, Mielgo, Blenis and Borst for the different cells used in this study. This work was supported by North West Cancer Research Grants CR1040 and CR1127 (to SV and GMC), Science Without Borders, CNPq 233624/2014-7, Ministry of Education, Brazil (to MM) and NIH Grants R03CA205496 and R01GM118437 (to XL).

Funding This work was supported by North West Cancer Research Grant CR1040, CR1127 (to SV and GMC), Science Without Borders, CNPq 233624/2014-7, Ministry of Education, Brazil (to MM) and NIH Grants R03CA205496 and R01GM118437 (to XL).

\section{Compliance with ethical standards}

Conflict of interest The authors declare that they have no conflict of interest.

Open Access This article is licensed under a Creative Commons Attribution 4.0 International License, which permits use, sharing, adaptation, distribution and reproduction in any medium or format, as long as you give appropriate credit to the original author(s) and the source, provide a link to the Creative Commons license, and indicate if changes were made. The images or other third party material in this article are included in the article's Creative Commons license, unless indicated otherwise in a credit line to the material. If material is not included in the article's Creative Commons license and your intended use is not permitted by statutory regulation or exceeds the permitted use, you will need to obtain permission directly from the copyright holder. To view a copy of this license, visit http://creativecommons. org/licenses/by/4.0/.

\section{References}

1. Kotschy A, Szlavik Z, Murray J, Davidson J, Maragno AL, Le Toumelin-Braizat G, et al. The MCL1 inhibitor S63845 is tolerable and effective in diverse cancer models. Nature. 2016;538:477-82.

2. Hanahan D, Weinberg RA. Hallmarks of cancer: the next generation. Cell. 2011;144:646-74.

3. Milani M, Byrne DP, Greaves G, Butterworth M, Cohen GM, Eyers PA, et al. DRP-1 is required for BH3 mimetic-mediated mitochondrial fragmentation and apoptosis. Cell Death Dis. 2017;8:e2552.

4. Youle RJ, Strasser A. The BCL-2 protein family: opposing activities that mediate cell death. Nat Rev Mol Cell Biol. 2008;9:47-59.

5. Oltersdorf T, Elmore SW, Shoemaker AR, Armstrong RC, Augeri DJ, Belli BA, et al. An inhibitor of Bcl-2 family proteins induces regression of solid tumours. Nature. 2005;435:677-81.

6. Quentmeier H, Drexler HG, Hauer V, MacLeod RAF, Pommerenke C, Uphoff CC, et al. Diffuse large B cell lymphoma cell line U-2946: model for MCL1 inhibitor testing. PLoS ONE. 2016;11: $\mathrm{e} 0167599$.

7. Tse C, Shoemaker AR, Adickes J, Anderson MG, Chen J, Jin S, et al. ABT-263: a potent and orally bioavailable Bcl-2 family inhibitor. Cancer Res. 2008;68:3421-8.

8. Del Gaizo Moore V, Brown JR, Certo M, Love TM, Novina CD, Letai A. Chronic lymphocytic leukemia requires BCL2 to sequester prodeath BIM, explaining sensitivity to BCL2 antagonist ABT-737. J Clin Invest. 2007;117:112-21.

9. Souers AJ, Leverson JD, Boghaert ER, Ackler SL, Catron ND, Chen J, et al. ABT-199, a potent and selective BCL-2 inhibitor, achieves antitumor activity while sparing platelets. Nat Med. 2013;19:202-8.

10. Roberts AW, Davids MS, Pagel JM, Kahl BS, Puvvada SD, Gerecitano JF, et al. Targeting BCL2 with venetoclax in relapsed chronic lymphocytic leukemia. N Engl J Med. 2015;374:311-22.

11. Luedtke DA, Niu X, Pan Y, Zhao J, Liu S, Edwards H, et al. Inhibition of Mcl-1 enhances cell death induced by the Bcl-2selective inhibitor ABT-199 in acute myeloid leukemia cells. Signal Transduct Target Ther. 2017;2:17012.

12. Leverson JD, Zhang H, Chen J, Tahir SK, Phillips DC, Xue J, et al. Potent and selective small-molecule MCL-1 inhibitors demonstrate on-target cancer cell killing activity as single agents and in combination with ABT-263 (navitoclax). Cell Death Dis. 2015;6:e1590.

13. Lin KH, Winter PS, Xie A, Roth C, Martz CA, Stein EM, et al. Targeting MCL-1/BCL-XL forestalls the acquisition of resistance to ABT-199 in acute myeloid leukemia. Sci Rep. 2016;6:27696.

14. Teh TC, Nguyen NY, Glaser S, Moujalled D, Pomilio G. Eradication of acute myeloid leukemia is enhanced by combined Bcl-2 and Mcl-1 targeting. Blood. 2014;124:988.

15. Pan R, Ruvolo VR, Wei J, Konopleva M, Reed JC, Pellecchia M, et al. Inhibition of Mcl-1 with the pan-Bcl-2 family inhibitor (-) BI97D6 overcomes ABT-737 resistance in acute myeloid leukemia. Blood. 2015;126:363-72.

16. Pan R, Hogdal LJ, Benito JM, Bucci D, Han L, Borthakur G, et al. Selective BCL-2 inhibition by ABT-199 causes on-target cell death in acute myeloid leukemia. Cancer Discov. 2014;4:362-75.

17. Inoue-Yamauchi A, Jeng PS, Kim K, Chen H-C, Han S, Ganesan YT, et al. Targeting the differential addiction to anti-apoptotic BCL-2 family for cancer therapy. Nat Commun. 2017;8:16078.

18. O'Neill KL, Huang K, Zhang J, Chen Y, Luo X. Inactivation of prosurvival Bcl-2 proteins activates Bax/Bak through the outer mitochondrial membrane. Genes Dev. 2016;30:973-88.

19. Takahashi H, Chen MC, Pham H, Matsuo Y, Ishiguro H, Reber $\mathrm{HA}$, et al. Simultaneous knock-down of Bcl-xL and Mcl-1 induces apoptosis through Bax activation in pancreatic cancer cells. Biochim Biophys Acta. 2013;1833:2980-7.

20. Zhang H, Guttikonda S, Roberts L, Uziel T, Semizarov D, Elmore $\mathrm{SW}$, et al. Mcl-1 is critical for survival in a subgroup of non-smallcell lung cancer cell lines. Oncogene. 2010;30:1963-8.

21. van Delft MF, Wei AH, Mason KD, Vandenberg CJ, Chen L, Czabotar PE, et al. The BH3 mimetic ABT-737 targets selective $\mathrm{Bcl}-2$ proteins and efficiently induces apoptosis via Bak/Bax if Mcl-1 is neutralized. Cancer Cell. 2006;10:389-99.

22. Lin X, Morgan-Lappe S, Huang X, Li L, Zakula DM, Vernetti LA, et al. "Seed" analysis of off-target siRNAs reveals an essential role of Mcl-1 in resistance to the small-molecule Bcl-2/ Bcl-XL inhibitor ABT-737. Oncogene. 2007;26:3972-9.

23. Vogler M, Butterworth M, Majid A, Walewska RJ, Sun X-M, Dyer MJS, et al. Concurrent up-regulation of BCL-XL and BCL2A1 induces approximately 1000-fold resistance to ABT-737 in chronic lymphocytic leukemia. Blood. 2009;113: 4403-13.

24. Varadarajan S, Vogler M, Butterworth M, Dinsdale D, Walensky LD, Cohen GM. Evaluation and critical assessment of putative MCL-1 inhibitors. Cell Death Differ. 2013;20:1475-84.

25. Lucas CM, Milani M, Butterworth M, Carmell N, Scott LJ, Clark RE, et al. High CIP2A levels correlate with an antiapoptotic phenotype that can be overcome by targeting BCL-XL in chronic myeloid leukemia. Leukemia. 2016;30:1273-81.

26. Delbridge AR, Aubrey BJ, Hyland C, Bernardini JP, Di Rago L, Garnier J-M, et al. The BH3-only proteins BIM and PUMA 
are not critical for the reticulocyte apoptosis caused by loss of the pro-survival protein BCL-XL. Cell Death Dis. 2017;8:e2914.

27. Varadarajan S, Poornima P, Milani M, Gowda K, Amin S, Wang H-G, et al. Maritoclax and dinaciclib inhibit MCL-1 activity and induce apoptosis in both a MCL-1-dependent and independent manner. Oncotarget. 2015;6:12668-81.

28. Varadarajan S, Butterworth M, Wei J, Pellecchia M, Dinsdale D, Cohen GM. Sabutoclax (BI97C1) and BI112D1, putative inhibitors of MCL-1, induce mitochondrial fragmentation either upstream of or independent of apoptosis. Neoplasia. 2013;15:568-78.

29. Wang C, Youle RJ. Predominant requirement of Bax for apoptosis in HCT116 cells is determined by Mcl-1's inhibitory effect on Bak. Oncogene. 2011;31:3177-89.

30. Hantusch A, Das KK, García-Sáez AJ, Brunner T, Rehm M. Bax retrotranslocation potentiates Bcl-xL's antiapoptotic activity and is essential for switch-like transitions between MOMP competency and resistance. Cell Death Dis. 2018;9:430.

31. Edlich F, Banerjee S, Suzuki M, Cleland MM, Arnoult D, Wang $\mathrm{C}$, et al. $\mathrm{Bcl}-\mathrm{x}(\mathrm{L})$ retrotranslocates Bax from the mitochondria into the cytosol. Cell. 2011;145:104-16.

32. Todt F, Cakir Z, Reichenbach F, Emschermann F, Lauterwasser J, Kaiser A, et al. Differential retrotranslocation of mitochondrial Bax and Bak. EMBO J. 2015;34:67-80.
33. Todt F, Cakir Z, Reichenbach F, Youle RJ, Edlich F. The C-terminal helix of Bcl-x $(\mathrm{L})$ mediates Bax retrotranslocation from the mitochondria. Cell Death Differ. 2013; $20: 333-42$.

34. Lauterwasser J, Todt F, Zerbes RM, Nguyen TN, Craigen W, Lazarou M, et al. The porin VDAC2 is the mitochondrial platform for Bax retrotranslocation. Sci Rep. 2016;6:32994.

35. Schellenberg B, Wang P, Keeble JA, Rodriguez-Enriquez R, Walker S, Owens TW, et al. Bax exists in a dynamic equilibrium between the cytosol and mitochondria to control apoptotic priming. Mol Cell. 2013;49:959-71.

36. Renault TT, Teijido O, Missire F, Ganesan YT, Velours G, Arokium $\mathrm{H}$, et al. Bcl-xL stimulates Bax relocation to mitochondria and primes cells to ABT-737. Int J Biochem Cell Biol. 2015;64:136-46.

37. Wu X, Zhang L-S, Toombs J, Kuo Y-C, Piazza JT, Tuladhar R, et al. Extra-mitochondrial prosurvival BCL-2 proteins regulate gene transcription by inhibiting the SUFU tumour suppressor. Nat Cell Biol. 2017;19:1226-36.

38. Escudero S, Zaganjor E, Lee S, Mill CP, Morgan AM, Crawford $\mathrm{EB}$, et al. Dynamic regulation of long-chain fatty acid oxidation by a noncanonical interaction between the MCL-1 BH3 helix and VLCAD. Mol Cell. 2018;69:729. e7 